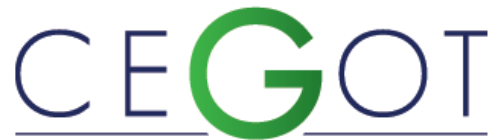

Centro de Estudos de Geografia e Ordenamento do Território
Geografia e Ordenamento do Território, Revista Eletrónica Centro de Estudos de Geografia e Ordenamento do Território http://cegot.org

MATTOS, KARINA

Universidade Estadual Paulista "Júlio de Mesquita Filho" UNESP Bauru/ Faculdade de Arquitetura, Artes e Comunicação

- FAAC/ Programa de Pós-Graduação em Arquitetura e Urbanismo - PPGARQ/ Centro Universitário de Lins

Av. Eng. Luís Edmundo Carrijo Coube, 14-01, 17033-360, Bauru, Brasil karina.mattos@unilins.edu.br

CONSTANTINO, NORMA

Universidade Estadual Paulista "Júlio de Mesquita Filho" -

UNESP Bauru/ Faculdade de Arquitetura, Artes e Comunicação

- FAAC/ Programa de Pós-Graduação em Arquitetura e

Urbanismo - PPGARQ/

Av. Eng. Luís Edmundo Carrijo Coube, 14-01, 17033-360, Bauru, Brasil

nconst@faac.unesp.br

\title{
Benefícios dos espaços verdes urbanos: valorização e avaliação pela população
}

Benefits of urban green spaces: valuation and evaluation by population

Referência: Mattos, Karina; Constantino, Norma (2019). Benefícios dos espaços verdes urbanos: valorização e avaliação pela população. Revista de Geografia e Ordenamento do Território (GOT), n. ${ }^{\circ} 16$ (março). Centro de Estudos de Geografia e Ordenamento do Território, p. 227-249, dx.doi.org/10.17127/got/2019.16.010

\section{RESUMO}

A importância dos espaços verdes para a conformação da paisagem, o ordenamento das cidades e a qualidade de vida dos cidadãos tem levado a sua crescente valorização. No entanto, a visão contemporânea baseada em sua multifuncionalidade tem trazido novos desafios ao planejamento urbano. Assim, entender como as diferentes instâncias da sociedade avaliam os benefícios associados aos espaços verdes urbanos torna-se fundamental para o desenvolvimento e aplicação de estratégias e políticas públicas de planejamento e gestão mais adequadas. Neste trabalho são explorados os resultados de uma pesquisa realizada com os moradores das cidades brasileiras de Botucatu e Bauru (SP), por meio do Best-Worst Scaling (BWS). Os resultados demonstram que os benefícios não são valorizados da mesma forma nas duas áreas urbanas, embora existam algumas semelhanças.

Palavras-chave: espaços verdes urbanos; benefícios dos espaços verdes urbanos; BestWorst Scaling (BWS); políticas públicas.

\section{ABSTRACT}

The importance of the green spaces for the conformation of the landscape, the planning of cities and citizens' life quality has led to their increasing valuation. However, the contemporary view based on their multifunctionality has brought new challenges to urban projects. So, to understand how different instances of society evaluate the benefits associated to the urban green spaces has been fundamental to development and 
application of more adequate strategies and public policy of planning and management. In this paper, it has been explored the results of a research carried out with Brazilian city residents of Botucatu and Bauru (SP), by the Best-Worst Scaling (BWS). The results have demonstrated that the benefits are not equally valued in both urban areas, although there have been some similarities.

Keywords: urban green spaces; benefits of urban green spaces; Best-Worst Scaling (BWS); public politics

\section{Introdução ${ }^{1}$}

Nos últimos anos, a valorização dos espaços verdes urbanos cresceu significativamente, apoiando-se na exposição de sua multifuncionalidade. Estudos que tratam de questões relativas a esses espaços partem da constatação de que as suas funções e os seus benefícios têm variado e evoluído ao longo da história, acompanhando as transformações das cidades e da sociedade. De fato, foram várias as funções ambientais, sociais e culturais desde as primeiras manifestações artísticas dos jardins, passando pelas áreas verdes de propriedades privadas para uso das classes privilegiadas até a criação no século XVIII de grandes zonas verdes para uso da população. Durante o século XIX, importantes ampliações urbanísticas foram responsáveis pela construção de avenidas arborizadas e grandes zonas verdes que permitiam a entrada de ar e luz. A partir desse período, os espaços verdes foram sendo exaltados por funções específicas de ordem estética, social e higienista. No entanto, durante a primeira metade do século $X X$, as mudanças ocorridas comprometeram de forma significativa a relação do homem com a cidade, afetando diretamente a qualidade do meio urbano e o bem-estar da população (Romero, 2007), suscitando a partir da segunda metade do século XX, desafios e preocupações ambientais globais. Com as crescentes preocupações e a necessidade de se desenvolver mecanismos de diminuição e contenção dos problemas ambientais nas cidades, a partir desse período os espaços verdes passaram a ter um certo destaque na esfera das políticas públicas (Madureira et al., 2014, p. 2311). Um caráter

\footnotetext{
${ }^{1}$ Este artigo baseia-se parcialmente no conteúdo apresentado na dissertação de Mestrado em Arquitetura e Urbanismo da autora, intitulada "Espaços verdes urbanos: análise multimétodos para a valorização", concluída em 2017 no Programa de Pós-Graduação em Arquitetura e Urbanismo, da Universidade Estadual Paulista (UNESP), Bauru, Brasil, a qual recebeu apoio da Pró-Reitoria de Pós-Graduação (PROPG-UNESP) e Coordenação de Aperfeiçoamento de Pessoal de Nível Superior (CAPES). O material apresentado no presente artigo não contempla todo o tema abordado na dissertação.
} 
multifuncional foi progressivamente sendo marcado nas diferentes abordagens desses espaços, refletindo, assim, suas diversas funções e benefícios.

Atualmente, os espaços verdes mostram-se peça fundamental dentro da conformação da paisagem e do ordenamento da cidade, uma vez que proporcionam benefícios ambientais, econômicos, estéticos, sociais, entre outros. Acredita-se em uma atuação efetiva desses espaços para atenuar os efeitos da aplicação de sistemas equivocados de desenvolvimento urbano, baseados principalmente em interesses econômicos. Nesse sentido, busca-se um meio mais perceptível a seus usuários, já que as estruturas construídas e a forma como os elementos estão organizados e conectados influenciam diretamente na qualidade de vida, nos impactos ambientais, na forma da população se relacionar, refletindo na manutenção da cultura e na identidade do local (Herzog, 2013, p. 99).

Algumas das funções ambientais dos espaços verdes, quando inseridos nas áreas urbanas, incluem a minimização dos efeitos da poluição atmosférica; a diminuição de ruídos; a redução da temperatura e estabilização da umidade do ar; a retenção de pó e de dióxido de carbono; percolação da água; auxílio da biodiversidade; controle da fauna e da flora; manutenção e criação de habitats; além da conexão do meio urbano com o meio natural e da restauração da paisagem urbana (Forman, 1995; Spirn, 1995; Hough, 1998; Romero, 2001; Pellegrino et al., 2006; Fálcon, 2007). Na ordem econômica, propiciam uma valorização do local; oferecem importantes produtos; incentivam a prática de atividades comerciais - fortalecendo a economia e o turismo - e proporcionam um controle de gastos por parte da administração pública, uma vez que evitam inúmeros reparos ambientais decorrentes de sua ausência (Fálcon, 2007; Baycan-Levent, Vreeker e Nijkamp, 2009). Na dimensão estética, contribuem para a imagem da cidade, oferecendo diferentes cores, formas, fragrâncias, movimentos, texturas, propiciando maior harmonia ao meio. Ademais, desempenham importantes funções sociais e culturais, assumindo considerável papel sobre a qualidade de vida da população. Além de atenuar os problemas ambientais que afetam diretamente a saúde física dos cidadãos, são responsáveis por levar bem estar, dar sentido de lugar e disponibilizar memórias por toda a vida, contribuindo para a saúde mental dos usuários do espaço. Assim, proporcionam o contato com a natureza; experiências multissensoriais; educação ambiental; promoção da saúde e do bem estar; incentivo à prática de atividades recreativas, lúdicas, culturais e contemplativas; além de interação e 
convívio social (Kaplan, S., 1995; Kaplan, R., 2001; Carman, 2005; Moore e Cosco, 2005; Dines et al., 2006; Thompson, 2007; Baycan-Levent, Vreeker e Nijkamp, 2009;).

Apesar dos aspetos positivos e da importância dos diversos benefícios dos espaços verdes, a sua multifuncionalidade tem trazido novos desafios à gestão urbana, uma vez que sua implantação exige uma definição com relação às prioridades de função a fim de qualificálos. Em algumas cidades brasileiras, e especificamente do centro-oeste paulista, é notória a dificuldade de ordenamento destes espaços junto à malha e à paisagem urbana, uma vez que existem espaços verdes cada vez mais precários e desconectados. A falta de atuação do poder público em ações de participação popular, fez com que a renúncia a esses espaços só aumentasse, mudando a forma da população se relacionar com as áreas verdes e resultando, consequentemente, no pouco aproveitamento de seus diversos benefícios. Enquanto os cidadãos demonstram uma percepção e interpretação baseada na realidade cotidiana, de características culturais e simbólicas, o poder público apoia-se em uma visão de planejamento urbano baseado em uma operação padrão que trata o espaço como um local "vazio", "auto-reprodutível" e "monofuncional" (Leite, 1996, p. 11). Segundo Jacobs (2000, p. 14) "é tolice planejar a aparência de uma cidade sem saber que tipo de ordem inata e funcional ela possui". Torna-se importante afirmar, portanto, que reconhecer as múltiplas funções e os benefícios dos espaços verdes urbanos é fundamental para um planejamento ordenado, contudo, é apenas uma das formas de se qualificar parte de uma grande infraestrutura verde. Assim, ao analisar um elemento da forma urbana, é imprescindível a compreensão das apropriações, representações e conflitos do homem sobre a paisagem. Os espaços verdes urbanos, bem como os demais espaços livres das cidades, precisam ser compreendidos também conforme as ações e as necessidades do usuário (Mazzei, Colesanti e Santos, 2007, p. 37), pois, é a partir da delimitação desse conjunto que se constituirá o espaço do cidadão no meio urbano. O usar, o ver e o sentir do indivíduo sobre o espaço tornam-se fundamentais para a tomada de consciência de cada local, de acordo com os contextos ambiental, cultural, econômico, social e urbano.

Nos últimos anos, diversas investigações foram realizadas em importantes regiões metropolitanas ao redor do mundo para se conhecer e avaliar as preferências da população em relação aos benefícios dos espaços verdes, como as pesquisas desenvolvidas por: Sanesi e Chiarello (2005), na Itália, Vesely (2007), na Nova Zelândia, Jim e Shan (2013), na China, 
Peckham, Duinker e Ordóñez (2013), no Canadá e Madureira et al. (2015) em Portugal e França. Para isso, foram utilizados diferentes materiais e métodos, dentre os quais destacam-se as observações, entrevistas, questionários e o método Best-Worst Scaling (BWS), com o objetivo de se desenvolver uma base comparativa importante entre a opinião dos usuários e os fatores sociais, culturais, territoriais e políticos. Isto posto, considerou-se pertinente desenvolver pesquisa desta natureza em cidades do Brasil. No entanto, além de levantar, analisar e comparar a opinião da população em relação à percepção e os benefícios associados a esses espaços, mostrou-se pertinente uma análise com os seus contextos político, social e urbano.

Dessa forma, o presente trabalho explora os resultados de uma pesquisa realizada com os moradores das cidades brasileiras de Botucatu e Bauru, ambas localizadas no Centro-Oeste do Estado de São Paulo, sobre os benefícios dos espaços verdes urbanos, considerando os diferentes contextos nos quais estão inseridos.

\section{Metodologia}

O processo metodológico estruturou-se em duas fases. Na primeira foram realizadas pesquisas bibliográfica e documental, estudo de caso, cartografia e entrevistas a fim de identificar as principais políticas públicas e as ações da administração municipal frente aos espaços verdes urbanos das cidades analisadas (Figura 1), considerando os seus contextos histórico, político, social e urbano ${ }^{2}$. Na segunda fase, direcionada à opinião da população a respeito da percepção e dos benefícios dos espaços verdes urbanos, foram utilizados questionários e o método Best-Worst Scaling (BWS) ${ }^{3}$.

\footnotetext{
2 Essas etapas não estão detalhadas no presente artigo.

${ }^{3}$ Para a fase da pesquisa junto a população brasileira, uma parceria foi firmada entre as pesquisadoras brasileiras e os pesquisadores portugueses Helena Madureira, Fernando Nunes, José A. Vidal Oliveira e Teresa Madureira. Assim, a estrutura do questionário é a mesma aplicada em cidades de Portugal e França. $\mathrm{O}$ artigo "Benefícios atribuídos aos espaços verdes urbanos pela população: resultados de um inquérito conduzido em Lisboa e no Porto" (Madureira et al., 2014) traz os resultados da pesquisa com os espaços verdes urbanos portugueses, e que serviram de base para a elaboração da pesquisa no Brasil.
} 


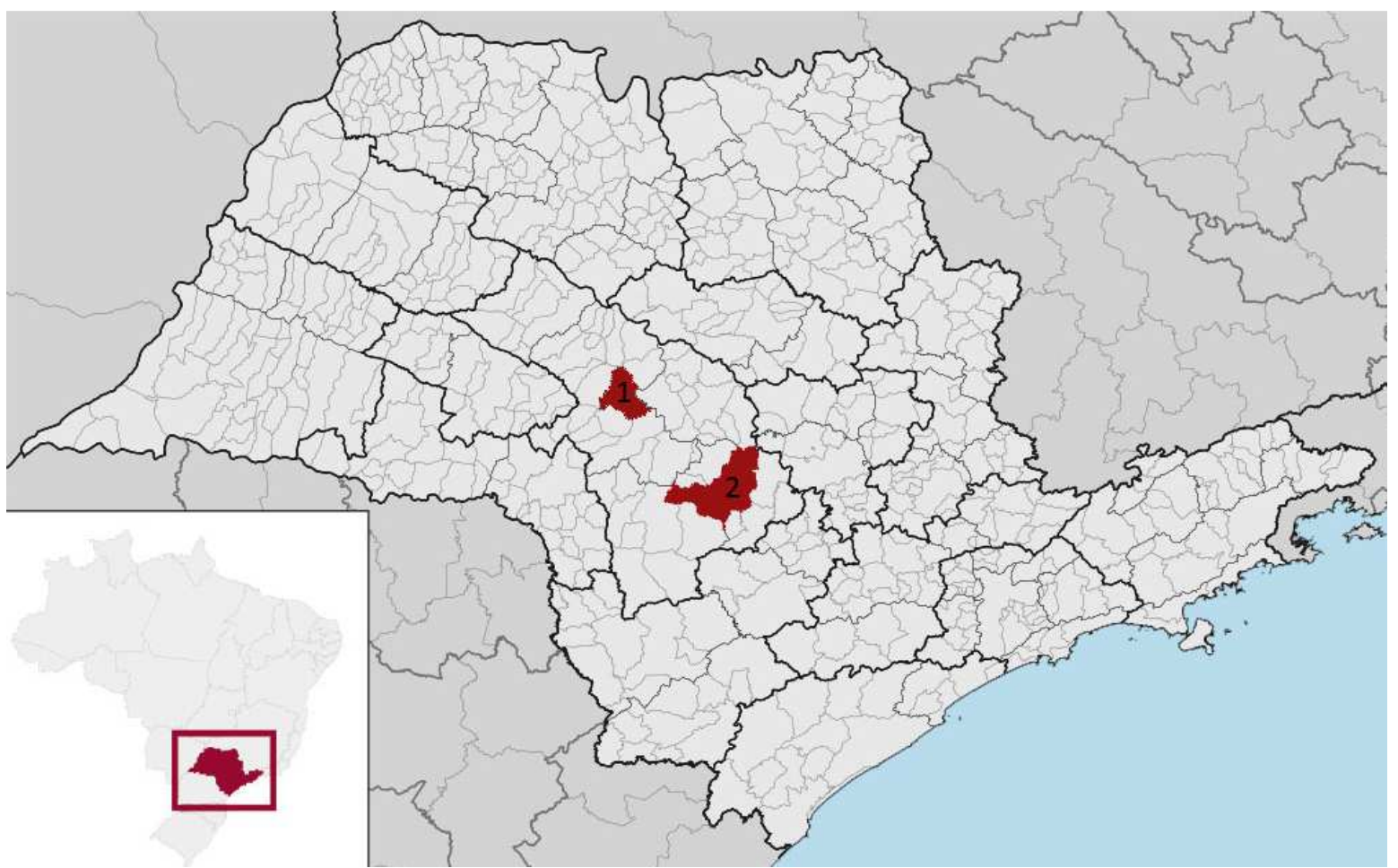

Figura 1 - Localização dos municípios de Bauru (1) e Botucatu (2) no Estado de São Paulo, Brasil Fonte: http://www.bibliotecavirtual.sp.gov.br/temas/sao-paulo/sao-paulo-aspectos-territoriais.php. Intervenção da autora

\subsection{Best-Worst Scaling (BWS)}

O método Best-Worst Scaling (BWS) foi introduzido pela primeira vez em 1988, por Jordan Louviere, da University of Alberta, em uma sessão da American Marketing Association's Advanced Research Techniques Forum (ART Forum), e aplicado em 1992, com o auxílio de Finn (Burke et al., 2013, p. 4). Em 1987, Louviere questionou-se sobre o que poderia fazer com as informações sobre o "least preferred" item de um conjunto de escolhas, além do tradicional "most preferred" item. Focado inicialmente em "objects", como marcas, atitudes, e metas de políticas públicas, o pesquisador iniciou os estudos com o método analisando o grau de preocupação que a população tinha com um conjunto de itens relacionados à segurança alimentar (Flynn e Marley, 2014, p. 03). Formalizado em 2005 por Marley e Louviere, o método permite hierarquizar o grau de importância de atributos. Os entrevistados são solicitados a identificar o benefício "mais" e "menos" importante dentro de um conjunto de atributos (De Magistris et al., 2011, p. 03), o que obriga o entrevistado a fazer escolhas e a refletir sobre suas respostas (Cunha, 2013, p. 29). Definidos os atributos a serem analisados, os mesmos são distribuídos em conjuntos chamados "sets", formados por 
três ou mais itens que surgem sequencialmente ao longo de um questionário (Madureira et al., 2014, p. 2313; Casini et al., 2008). O processo simultâneo de escolha dos atributos que apresentam maior diferença de valor é conhecido como MaxDiff (Sawtooth Software, 2013). Por meio da gestão de softwares apropriados, é possível assegurar que todos os atributos apareçam o mesmo número de vezes nos conjuntos aplicados (Madureira et al., 2014, p. 2313).

Inúmeras são as vantagens do BWS, dentre as quais destacam-se o uso de um intervalo de escala unidimensional de importância de atributos; maior poder de discriminação para a medida do grau de importância dada pelos entrevistados aos atributos e melhor combinação entre os benefícios; a prevenção de problemas de viés de classificação; assim como o fácil entendimento pelos entrevistados (Casini et al., 2008, p.4; De Magistris et al., 2011, p. 3). Para Cohen e Orme (2004), o método pode ser considerado uma extensão do Método de Comparação Pareada, mas com uma estrutura de questionamento mais eficiente (Casini et al., 2008, p. 4). De acordo com os responsáveis pelo novo método, nas escalas de avaliação utilizadas em pesquisas quantitativas, não existe a discriminação de itens pelo entrevistado, o que permite afirmar a importância simultânea de diversos itens. Além disso, a validade das escalas de avaliação e a interpretação da classificação dos valores é uma ação difícil e de confiabilidade desconhecida. "What does 7 out of 10 mean in terms of real life choices?". Dessa forma, o Best-Worst Scaling é apresentado em contrapartida a outras categorias de escala de avaliação (Flynn e Marley, 2014, p. 03).

\subsection{Universo e amostra}

Com base nos dados do Censo Demográfico 2010 do Instituto Brasileiro de Geografia e Estatística (IBGE, 2010), foram obtidas informações sobre gênero, idade, educação, rendimento e ocupação de cada população estudada. Assim, o Universo da investigação constituiu-se pela população residente nas cidades de Botucatu e Bauru, com 15 ou mais anos de idade (Tabela 1). 


\begin{tabular}{lcc}
\hline & Universo & \\
\hline & Botucatu & Bauru \\
\hline Homens & 48.003 & 131.394 \\
\hline Mulheres & 52.558 & 143.626 \\
\hline Total & 100.561 & 275.020 \\
\hline
\end{tabular}

Tabela 1 - Universo da investigação de Botucatu e Bauru

A amostra foi definida em $250^{4}$ indivíduos por município, distribuída por quotas, com variáveis de controle gênero e grupo de idade. A tabela 2 apresenta a amostra obtida (amostra real) ${ }^{5}$ para cada uma das cidades.

\begin{tabular}{|c|c|c|c|}
\hline & \multicolumn{3}{|c|}{ Amostra Obtida } \\
\hline & & Botucatu & Bauru \\
\hline \multicolumn{4}{|c|}{ Homens } \\
\hline \multirow{4}{*}{$\begin{array}{l}\frac{0}{0} \\
\frac{0}{0} \\
\text { O }\end{array}$} & 15-34 anos & 53 & 53 \\
\hline & $35-54$ anos & 41 & 42 \\
\hline & $\geq 55$ anos & 26 & 25 \\
\hline & Mulheres & & \\
\hline \multirow{4}{*}{$\begin{array}{l}\frac{0}{0} \\
\frac{\pi}{0}\end{array}$} & 15-34 anos & 53 & 53 \\
\hline & 35-54 anos & 44 & 45 \\
\hline & $\geq 55$ anos & 33 & 32 \\
\hline & Total & 250 & 250 \\
\hline
\end{tabular}

Tabela 2 - Composição da Amostra Obtida

Com relação às duas variáveis cruzadas, a amostra obtida iguala-se à amostra teórica. Dessa forma, a amostra adquirida (real ou obtida) é representativa quanto ao gênero e faixa etária.

\subsection{Procedimento de amostragem}

A investigação com a população foi conduzida online e divulgada por e-mails, redes sociais, jornais eletrônicos, páginas da internet e abordagens em locais públicos com wi-fi. Recorreu-se ao procedimento de amostragem não aleatória misto - amostragem por conveniência e quotas - aquela em que o participante coopera com a pesquisa em função de sua disponibilidade e vontade. Adotou-se a técnica de recrutamento por "snowball" ou

\footnotetext{
${ }^{4}$ Valor máximo admitido para a versão do Sawtooth Software, utilizado no estudo.

${ }^{5} \mathrm{~A}$ amostra teórica (representativa do universo) é a mesma da amostra obtida (amostra real).
} 
"bola de neve", em que os indivíduos que participam da pesquisa convidam outros participantes da sua rede de amigos, garantindo que a amostra cresça conforme o contato entre os envolvidos.

\subsection{Elaboração e aplicação do questionário e o Best-Worst Scaling (BWS)}

O questionário foi estruturado em três partes, assim como nas investigações de Portugal e França, contudo, com algumas adequações ao contexto brasileiro. Um texto de apresentação explicando a parceria entre as instituições, os objetivos, o conceito de espaços verdes urbanos como "áreas com vegetação no interior da cidade, públicas ou privadas, incluindo espaços com árvores, arbustos, flores ou gramados" e algumas questões pertinentes à ética da pesquisa foi apresentado previamente.

Na primeira parte foram inseridas questões relativas à caracterização socioeconômica da população, como idade, gênero, rendimento líquido mensal, nível de formação acadêmica, posição face ao trabalho, código postal da residência e do trabalho, e presença de menores de 15 anos vivendo na mesma residência que o entrevistado. A segunda parte incluiu questões sobre a percepção dos participantes em relação aos espaços verdes urbanos da cidade, como a presença de jardim na residência, a presença de espaços verdes a aproximadamente cinco minutos a pé da residência e do local de trabalho, a frequência de utilização de jardins e parques públicos e a classificação de satisfação geral em relação aos espaços verdes da cidade e da vizinhança. A terceira parte teve como objetivo avaliar a importância atribuída a dez diferentes benefícios dos espaços verdes urbanos por meio do Best-Worst Scaling, sendo portanto, a parte mais importante da investigação. Para essa etapa foram selecionados cinco benefícios ambientais - diminuir a poluição do ar da cidade, diminuir a temperatura do ar na cidade, reter o dióxido de carbono, contribuir para a diversidade e atenuar o ruído na cidade - e cinco socioculturais - aumentar a qualidade visual da cidade, favorecer o contato com a natureza, fomentar atividades recreativas e desportivas ao ar livre, promover a saúde e o bem-estar e facilitar o convívio e a interação social.

Os benefícios foram inseridos no Sawtooth Software e distribuídos em conjuntos. Cada conjunto era composto por quatro benefícios. Através da gestão do software foi possível 
garantir que os atributos aparecessem aproximadamente o mesmo número de vezes no total dos dez conjuntos apresentados e em primeiro, segundo, terceiro e quarto lugar (Sawtooth Software, 2013). Antes de iniciar a etapa, um texto explicou que as funções poderiam se repetir, sendo esta uma característica do método, e que os respondentes deveriam, dentro dos quatro benefícios de cada conjunto, escolher o "mais importante" e o "menos importante", salientando que o mesmo nunca poderia ser escolhido simultaneamente em um determinado conjunto. Cada participante avaliou cada um dos diferentes conjuntos de atributos apresentados e indicou o par que refletia a diferença máxima de importância. Foram realizados dois testes com a finalidade de certificar-se de que todos os ajustes estavam condizentes ao contexto brasileiro e que o software não apresentava problemas. O questionário ficou disponível na internet entre julho e outubro de 2016.

\subsection{Indicadores e análise dos resultados}

A análise dos resultados segue a estrutura do questionário e foi organizada em três seções principais. A primeira aborda as características dos entrevistados, por meio de análises descritivas e tabelas. A segunda foca na percepção dos espaços verdes urbanos da cidade através de frequências e percentuais. Para finalizar, a terceira etapa apresenta a preferência da população em relação aos benefícios dos espaços verdes urbanos. Por meio dos indicadores de análise ${ }^{6}$, obteve-se um ranking ordinal dos atributos, em uma escala de intervalo. Primeiramente, calculou-se a pontuação standard (best-worst) ${ }^{7}$ individuais para cada atributo. Para facilitar a interpretação dos dados, as pontuações best-worst foram transformadas em uma escala positiva, muito aproximada de uma escala de rácio ${ }^{8}$ de

\footnotetext{
${ }^{6} \mathrm{Na}$ análise de resultados podem ser utilizados vários indicadores, como Pontuação standard (best-worst); Raw score; Rescaled scores ou B/W rating e Share of importance. Abordou-se os indicadores Pontuação standard (best-worst) e Rescaled scores.

${ }^{7}$ A pontuação standard (best-worst), a nível agregado, é resultado da diferença entre o número de vezes que cada atributo é escolhido como mais e menos importante dividido pela dimensão da amostra. É a média do score B-W para cada atributo e pode ser interpretado como o número médio de vezes que o atributo foi escolhido como mais ou menos importante. Os diferentes atributos têm pontuações positivas e negativas que somam zero.

${ }^{8} \mathrm{~A}$ escala de rácio (ou proporcionais) faz parte de um conjunto de quatro tipos de escalas primárias de medida: escalas nominais; escalas ordinais; escalas de intervalos; e escalas de rácio. A escala de rácio apresenta o ponto zero absoluto (ausência da característica a medir). Com o rácio de medida, só a unidade
} 
probabilidade que varia de 0 a 100, passando para Rescaled Scores ${ }^{9}$ (Sawtooth Software, 2013). Com a soma dos itens analisados igual a 100 é possível determinar o percentual de vezes que o atributo foi escolhido quando comparado com os outros (Madureira et al., 2015). Para todas as etapas foi realizado também o teste "Qui-quadrado" para determinar a significância da relação entre as diferentes variáveis do estudo ${ }^{10}$.

\section{Resultados e Discussões}

Para uma análise comparativa ponderou-se as amostras, restituindo o peso de cada cidade no total ${ }^{11}$, observando que a dimensão da amostra nos dois centros urbanos foi a mesma (250 entrevistas), mesmo as cidades tendo universos com diferentes dimensões (100.561 indivíduos em Botucatu e 275.020 indivíduos em Bauru). Assim, considera-se uma amostra ponderada de 134 para Botucatu e 366 para Bauru, totalizando 500 participantes.

\subsection{Características das amostras}

As características socioeconômicas dos entrevistados dos dois centros urbanos podem ser comparadas na Tabela 3, por meio de seis variáveis. Com relação à idade e ao gênero constata-se que não existem diferenças significativas entre as modalidades nas duas cidades, assim como ocorre com o rendimento, se analisadas suas seis modalidades de classificação. Contudo, ao agrupá-las em duas - até $R \$ 3.000,00$ reais $^{12}$ e mais de $R \$ 3.000,00$ reais - observa-se uma diferença significativa. Em Botucatu $64 \%$ dos participantes tem rendimento mensal até $\mathrm{R} \$ 3.000,00$ reais e em Bauru 54\%. Quanto à ocupação, os ativos representam a maioria dos entrevistados tanto em Botucatu (73\%) quanto em Bauru (66\%), com uma predominância de assalariados em ambas as cidades. No que se refere à

de medida ou distância pode ser atribuída arbitrariamente. Uma vez determinada esta, todos os outros valores estarão definidos, podendo-se calcular rácios de valores de escala.

${ }^{9}$ Os rescaled scores resultam da transformação dos raw scores em probabilidades para os dados iniciais, sendo sua soma igual a 100 e são calculados diretamente pelo Sawtooth Software. Ver mais em Sawtooth Software Inc., 2013.

${ }^{10}$ Serão consideradas relações significativas aquelas que apresentam $p<0,05$.

${ }^{11}$ Os fatores de ponderação utilizados foram de 0,536 para os questionários de Botucatu e 1,464 para os de Bauru.

${ }^{12}$ Valor equivalente a aproximadamente 688,00 euros (referente a cotação de 1 euro=4,36 reais do dia 30 de julho de 2018). 
educação, há uma diferença expressiva entre os níveis de formação acadêmica dos participantes das diferentes localidades. Em Bauru, a maioria dos participantes têm ensino superior (72\%), diferente de Botucatu, onde 52\% dos participantes não têm. Essa diferença pode estar relacionada a fatores como a divulgação online da pesquisa, a falta de acesso ao questionário, o interesse desse grupo em participar e a presença maior de estudantes universitários em Bauru.

\begin{tabular}{|c|c|c|c|}
\hline \multicolumn{2}{|c|}{ Variáveis socioeconômicas } & \multirow{2}{*}{$\begin{array}{c}\text { Botucatu } \\
20,8 \%\end{array}$} & \multirow{2}{*}{$\begin{array}{l}\text { Bauru } \\
20,4 \%\end{array}$} \\
\hline \multirow{6}{*}{ Idade } & $15-24$ & & \\
\hline & $25-34$ & $21,6 \%$ & $22 \%$ \\
\hline & $35-44$ & $18 \%$ & $18,4 \%$ \\
\hline & $45-54$ & $16 \%$ & $16,4 \%$ \\
\hline & $55-64$ & $11,6 \%$ & $11,2 \%$ \\
\hline & $\geq 65$ & $12 \%$ & $11,6 \%$ \\
\hline \multirow{2}{*}{ Gênero } & Masculino & $48 \%$ & $48 \%$ \\
\hline & Feminino & $52 \%$ & $52 \%$ \\
\hline \multirow{6}{*}{ Rendimento* (reais) } & $<1.500$ & $32 \%$ & $23,6 \%$ \\
\hline & $1.500-3.000$ & $32,4 \%$ & $30,4 \%$ \\
\hline & $3.000-4.500$ & $13,2 \%$ & $15,2 \%$ \\
\hline & $4.500-6.000$ & $8 \%$ & $9,2 \%$ \\
\hline & $6.000-7.500$ & $4,8 \%$ & $5,6 \%$ \\
\hline & $\geq 7.500$ & $9,6 \%$ & $16 \%$ \\
\hline \multirow{5}{*}{ Educação* } & Ensino fundamental & $8 \%$ & $9,2 \%$ \\
\hline & Ensino médio incompleto & $6 \%$ & $1,2 \%$ \\
\hline & Ensino médio completo & $27,2 \%$ & $13,2 \%$ \\
\hline & Curso Técnico & $10,4 \%$ & $4,4 \%$ \\
\hline & Ensino superior & $48,4 \%$ & $72 \%$ \\
\hline \multirow{10}{*}{ Ocupação } & Empresário & $10 \%$ & $3,6 \%$ \\
\hline & Prof. liberal/conta própria & $14,8 \%$ & $13,6 \%$ \\
\hline & Trabalhador empregado & $47,2 \%$ & $47,6 \%$ \\
\hline & Outros ativos & $1,2 \%$ & $1,6 \%$ \\
\hline & Desempregado & $2,8 \%$ & $0,4 \%$ \\
\hline & À procura do $1^{\circ}$ emprego & $0,8 \%$ & $1,2 \%$ \\
\hline & Estudante & $7,2 \%$ & $16,8 \%$ \\
\hline & Doméstica & $1,2 \%$ & $0,8 \%$ \\
\hline & Aposentado & $14 \%$ & $13,2 \%$ \\
\hline & Outros não ativos & $0,8 \%$ & $1,2 \%$ \\
\hline \multirow{2}{*}{ Crianças* } & $\operatorname{Sim}$ & $30,4 \%$ & $19,6 \%$ \\
\hline & Não & $69,6 \%$ & $80,4 \%$ \\
\hline \multicolumn{2}{|l|}{ Amostra Ponderada } & 134 (100\%) & 366 (100\%) \\
\hline \multicolumn{2}{|l|}{ Amostra Teórica } & 250 (100\%) & $250(100 \%)$ \\
\hline
\end{tabular}

As variáveis socioeconômicas assinaladas com $\left({ }^{*}\right)$ são as que apresentam diferenças significativas a nível das cidades $(p<0,05)$.

Tabela 3 - Comparação entre as características socioeconômicas dos entrevistados de Botucatu e Bauru 


\subsection{A população e os espaços verdes urbanos}

A percepção geral dos participantes sobre os espaços verdes urbanos pode ser comparada a partir dos três grupos de perguntas desenvolvidos na pesquisa, que envolvem diferentes escalas da cidade - a percepção da proximidade a jardins, a frequência de visitas a jardins públicos e a satisfação com os espaços verdes da cidade. Com relação à presença de jardim na residência, constata-se uma diferença significativa na resposta dos participantes. Enquanto 53\% dos entrevistados residentes em Bauru têm um espaço verde dentro do local onde moram, em Botucatu representam 43\%. Considerando o total de participantes, os valores equiparam-se em $50 \%$. Na avaliação sobre a percepção da presença de jardim ou parque público a aproximadamente 5 minutos a pé da residência e do trabalho, no entanto, não existem diferenças significativas. Os resultados mostram que a maioria dos participantes afirma ter acesso a esses locais em pouco tempo da residência - $77 \%$ em Botucatu e 74\% em Bauru - e do trabalho - 70\% em Botucatu e 66\% em Bauru ${ }^{13}$ (Figura 2).

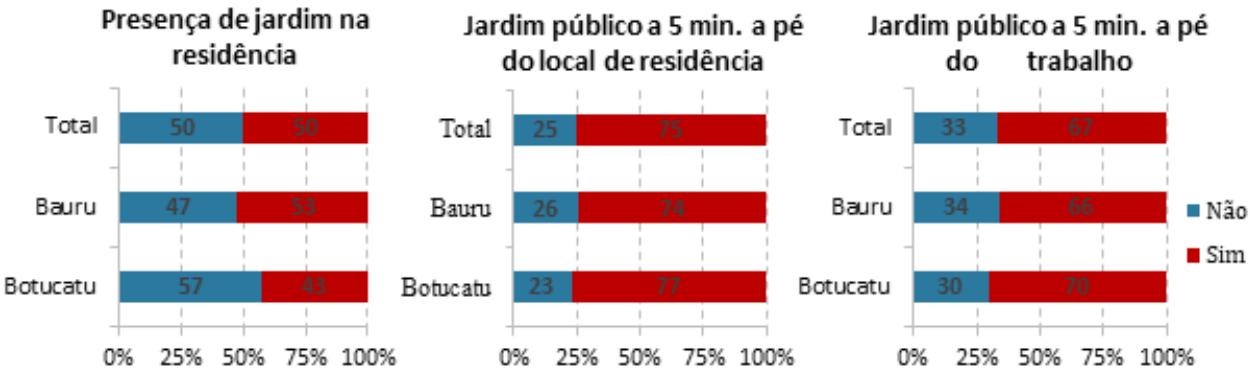

Figura 2 - Presença de jardim da residência, jardim público a 5 minutos a pé do local de residência e de trabalho

Três principais constatações surgem destas primeiras questões. Primeiro, os espaços verdes poderiam estar mais presentes nas residências dos participantes, de ambas as cidades, sendo portanto, mais valorizados na escala do lote. Segundo, os participantes de Botucatu têm uma facilidade um pouco maior de acesso a jardins e parques públicos. Terceiro, os espaços verdes localizam-se mais próximo das residências do que dos locais de trabalho dos participantes.

Os resultados referentes à frequência de visitas a jardins mostram diferenças entre os dois meios urbanos (Figura 3). De um modo geral, são poucos os que utilizam esses espaços, já

\footnotetext{
${ }^{13}$ Quanto a proximidade do local de trabalho, houve uma considerável taxa de não resposta à questão. A ausência de respondentes pode estar relacionada a diversos fatores, como má formulação ou má entendimento da questão e a ausência de um local fixo de trabalho.
} 
que apenas $36 \%$ dos participantes deslocam-se uma ou mais vezes por semana a jardins e parques públicos. Os residentes entrevistados de Botucatu, no entanto, frequentam mais que os de Bauru - cerca de $48 \%$ deslocam-se semanalmente, contra $32 \%$, respectivamente.

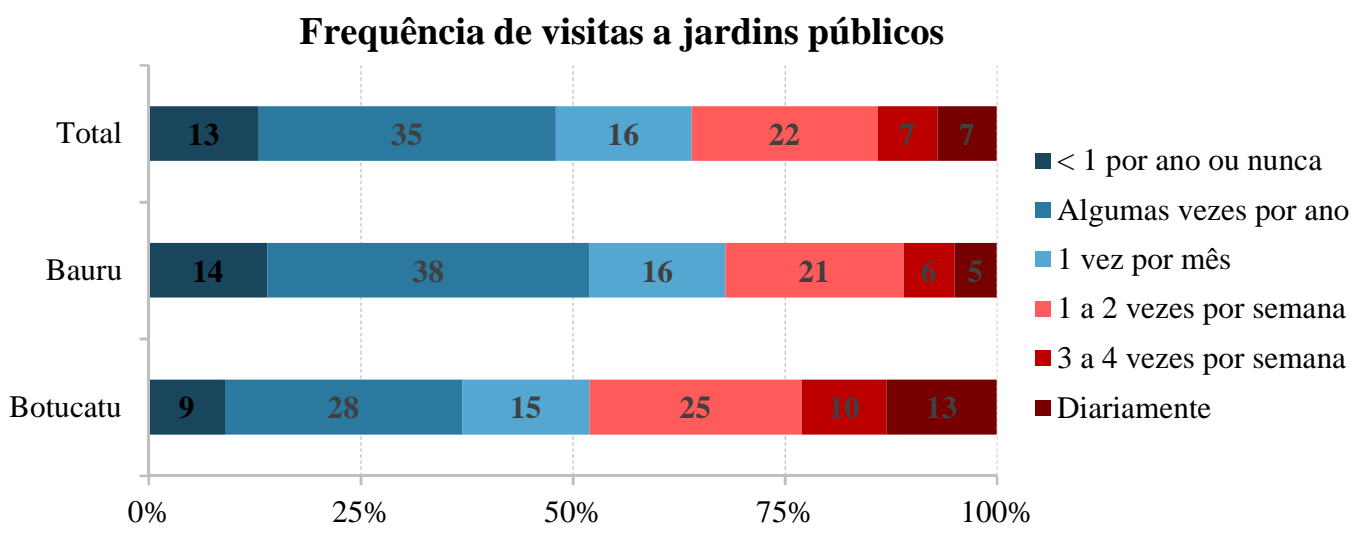

Figura 3 - Frequência de visitas a jardins e parques públicos

Quanto à satisfação com os espaços verdes da cidade e na proximidade da residência, há expressivas divergências entre a opinião dos participantes de Botucatu e Bauru. Enquanto a maioria de Botucatu mostra-se satisfeita ou muito satisfeita com os espaços verdes da cidade e próximo ao local onde moram, a maioria de Bauru mostra-se insatisfeita ou muito insatisfeita. Constata-se ainda que em Botucatu a satisfação com espaços verdes na proximidade da residência é menor do que com os espaços da cidade, já em Bauru a avaliação é o contrário (Figuras 4 e 5). Considerando todos os participantes, constata-se uma insatisfação de $51 \%$ para os espaços verdes da cidade ( $24 \%$ em Botucatu e $61 \%$ em Bauru) e $50 \%$ para próximos a residência (34\% em Botucatu e 56\% em Bauru). Em uma escala de 1 a 5 , onde 1 é muito insatisfeito e 5 muito satisfeito, o valor médio obtido para o total das cidades é 2,72 para os espaços verdes da cidade e 2,77 para os próximos da residência, ambos abaixo do ponto médio 3. No entanto, é importante destacar que esses valores variam de cidade para cidade. 


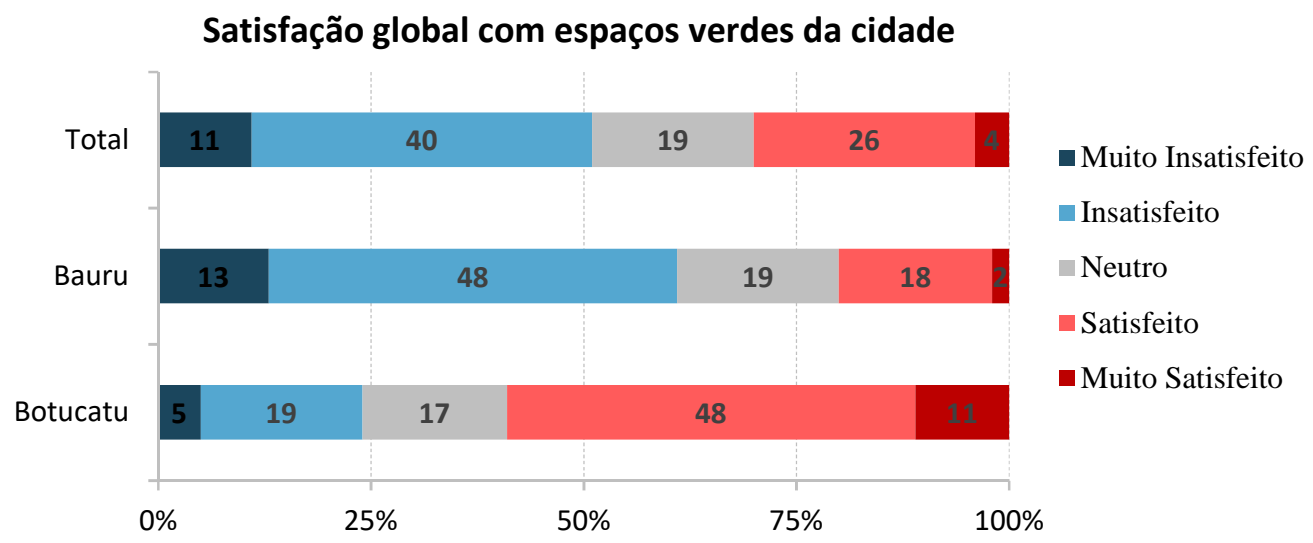

Figura 4 - Nível de satisfação dos entrevistados com os espaços verdes das cidades

\section{Satisfação global com espaços verdes próximo a residência}

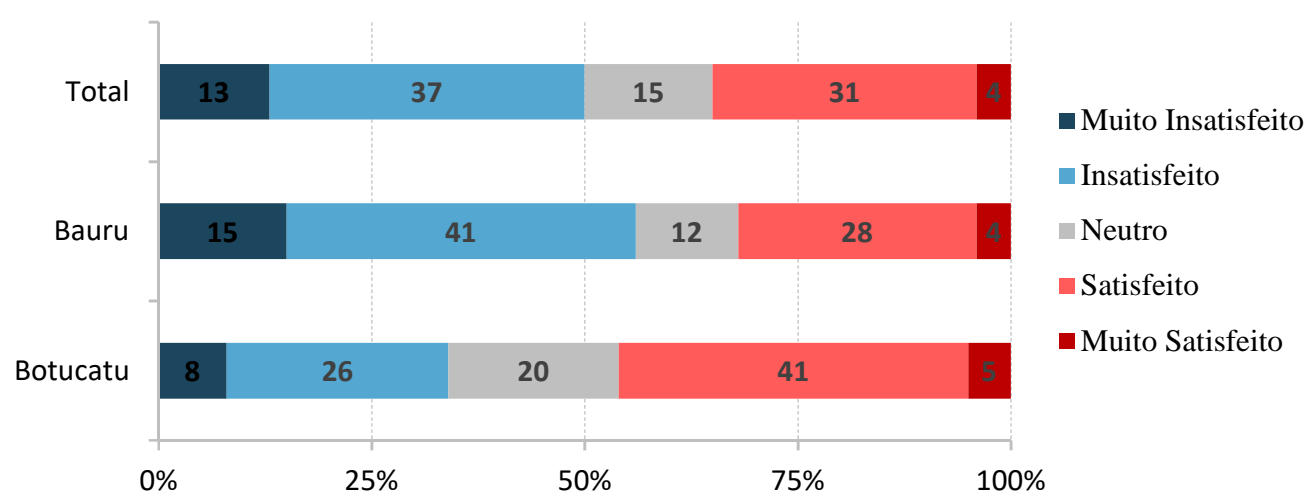

Figura 5 - Nível de satisfação dos entrevistados com os espaços verdes próximo a residência

A partir dos resultados apresentados, constata-se que em Botucatu, onde foram observadas ações mais diretas da administração pública para a criação e conservação de espaços verdes, a frequência de visitas e os níveis de satisfação dos entrevistados mostram-se mais positivos do que em Bauru, onde verificou-se espaços verdes sem tratamento adequado e uma gestão ambiental não tão efetiva.

\subsection{A importância dos benefícios ambientais e socioculturais para os cidadãos}

No que se refere a importância dos benefícios ambientais e socioculturais dos espaços verdes urbanos para a população, observa-se os "Rescaled Scores" pertencentes aos dez benefícios avaliados pelos participantes na Tabela 4. Na figura 6 foram hierarquizados os valores do indicador de acordo com os resultados gerais. 


\begin{tabular}{lccc}
\hline Atributo & Botucatu & Bauru & Total \\
& RsS & RsS & RsS \\
\hline Diminuir a poluição do ar da cidade & 15,4 & 14,6 & 14,8 \\
Diminuir a temperatura do ar na cidade* & 9,3 & 11,6 & 11,0 \\
Reter o dióxido de carbono* & 12,7 & 9,9 & 10,6 \\
Contribuir para a biodiversidade* & 11,3 & 9,5 & 10,0 \\
Atenuar o ruído na cidade* & $\mathbf{2 , 3}$ & 1,6 & 1,8 \\
\hline Atributos Ambientais & $\mathbf{5 1}$ & $\mathbf{4 7 , 2}$ & $\mathbf{4 8 , 2}$ \\
\hline Aumentar a qualidade visual da cidade & 4,3 & 4,9 & 4,7 \\
Favorecer o contato com a natureza & 11,8 & 12,4 & 12,3 \\
Fomentar atividades recreativas e desportivas ao ar livre & 9,8 & 10,7 & 10,5 \\
Promover a saúde e o bem-estar & 16,9 & $\mathbf{1 7 , 2}$ & 17,1 \\
Facilitar o convívio e a interação social & 6,2 & $\mathbf{7 , 6}$ & $\mathbf{7 , 2}$ \\
\hline Atributos Sociais e Culturais & $\mathbf{4 9}$ & $\mathbf{5 2 , 8}$ & $\mathbf{5 1 , 8}$ \\
\hline Total & $\mathbf{1 0 0 , 0}$ & $\mathbf{1 0 0 , 0}$ & $\mathbf{1 0 0 , 0}$
\end{tabular}

RsS: "Rescaled Scores"

Os atributos assinalados com $(*)$ são os que apresentam diferenças significativas a nível das cidades $(p<0,05)$. Tabela 4 - "Rescaled Scores"

Considerando o resultado total, verifica-se que os benefícios sociais e culturais $(51,8 \%)$ dos espaços verdes urbanos são um pouco mais valorizados do que os benefícios ambientais $(48,2 \%)$, havendo quase que uma igualdade entre os dois conjuntos. Entre os três benefícios mais valorizados - "promover a saúde e o bem-estar" $(17,1 \%)$, "diminuir a poluição do ar da cidade" $(14,8 \%)$ e "favorecer o contato com a natureza" (12,3\%) - também existe uma alternância entre os grupos, com uma pequena prevalência dos benefícios socioculturais.

Entre os atributos valorizados, mas de forma mais moderada, estão "diminuir a temperatura do ar na cidade" (11\%) e "reter o dióxido de carbono" (10,6\%). "Fomentar atividades recreativas e desportivas ao ar livre" $(10,5 \%)$ e "contribuir para a biodiversidade" (10\%) são considerados menos importantes, contudo também de forma moderada, quando comparados aos três últimos menos importantes - "facilitar o convívio e a interação social" $(7,2 \%)$, "aumentar a qualidade visual da cidade" $(4,7 \%)$ e "atenuar o ruído na cidade" $(1,8 \%)$. 


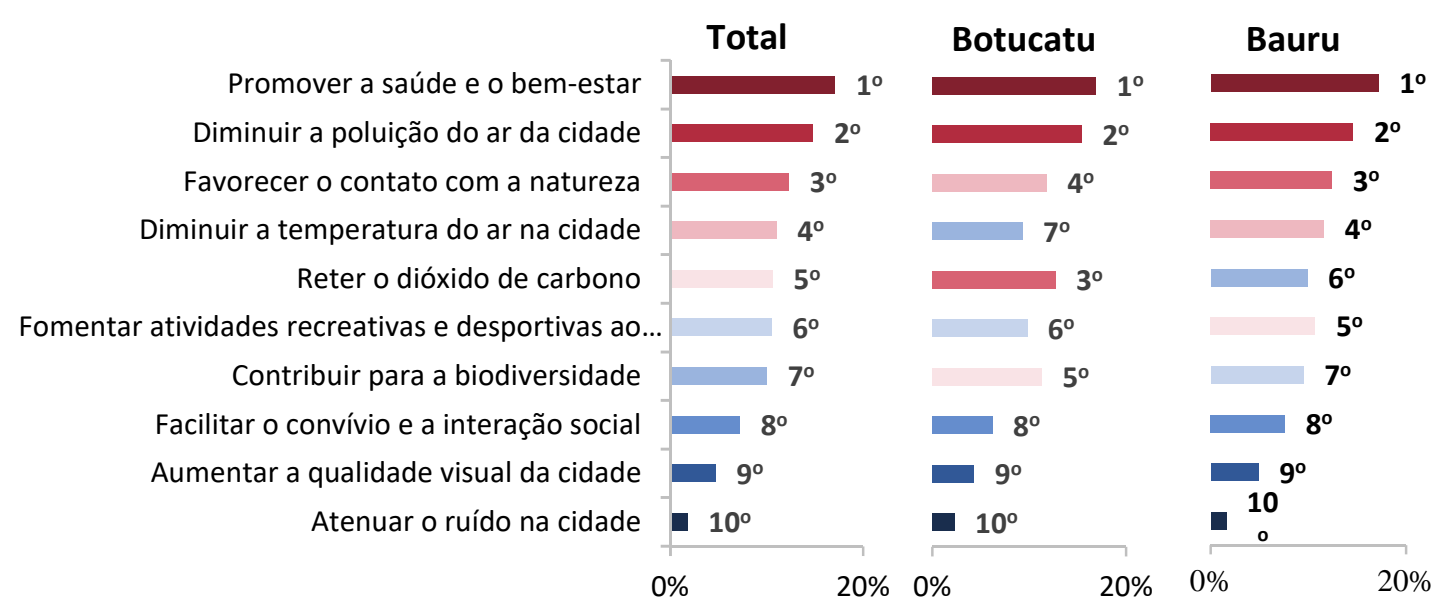

Figura 6 - "Rescaled Scores" das cidades hierarquizados com base nos "Rescaled Scores" geral

Ao analisar a relação entre os indicadores de cada atributo por cidade, constatam-se algumas semelhanças e diferenças entre os centros urbanos. Quanto aos benefícios dos espaços verdes mais importantes, é unânime entre os participantes a valorização de "promover a saúde e bem-estar" e "diminuir a poluição do ar da cidade", como primeiro e segundo lugar, respectivamente. Há um consenso também no posicionamento hierárquico dos benefícios menos valorizados, que são "facilitar o convívio e a interação social" $\left(8^{\circ}\right)$, "aumentar a qualidade visual da cidade" $\left(9^{\circ}\right)$ e "atenuar o ruído na cidade" $\left(10^{\circ}\right)$, embora exista uma diferença significativa entre os indicadores de importância para esse último atributo a nível das cidades.

Essas diferenças significativas entre os indicadores de importância se mantém para os benefícios ambientais "diminuir a temperatura do ar da cidade", "reter o dióxido de carbono" e "contribuir para a biodiversidade". Quanto a hierarquização dos mesmos, observa-se que enquanto "diminuir a temperatura do ar da cidade" ocupa apenas a $7^{\circ}$ posição em Botucatu, no ranking de Bauru e no total é considerado mais importante, ocupando a $4^{\circ}$ posição. O benefício "reter o dióxido de carbono" ocupa diferentes posições, em cada um dos rankings. Em Botucatu é um dos mais importantes, na $3^{\circ}$ posição, já em Bauru e no total, sua valorização é menor, ocupando a $6^{\circ}$ e a $5^{\circ}$ posição, respectivamente. "Contribuir para a biodiversidade" é o $5^{\circ}$ benefício mais importante para os participantes de Botucatu, sendo de forma moderada um dos menos importantes para os participantes de Bauru e no total, ocupando a $7^{\circ}$ posição. 
Para os demais benefícios, há variações hierárquicas, mas não existem diferenças significativas entre os indicadores a nível das cidades. "Favorecer o contato com a natureza" muda da $4^{\circ}$ posição em Botucatu para a $3^{\circ}$ em Bauru e no total, permanecendo entre as mais importantes e "fomentar atividades recreativas e desportivas ao ar livre" de $6^{\circ}$ em Botucatu e no total, sobe para a $5^{\circ}$ posição em Bauru.

A partir da comparação entre os dados levantados, constata-se que os benefícios não são igualmente classificados pelas populações de Botucatu e Bauru, apesar de existir um consenso entre os participantes das duas cidades no que se refere aos benefícios mais e menos importantes. As semelhanças e incompatibilidades na classificação não permitem afirmar, dessa forma, se as divergências e influências locais interferem na hierarquização, uma vez que mesmo com contextos ambientais, políticos e sociais diferentes, alguns benefícios foram igualmente valorizados e menos valorizados. No entanto, detecta-se que fatores específicos relacionados a esses contextos surgem em meio a classificação. Ao analisar os rankings de Botucatu e Bauru, por exemplo, verifica-se que há uma diferença entre a valorização do benefício "diminuir a temperatura do ar da cidade" nos dois centros. Enquanto em Botucatu, onde as temperaturas são mais amenas em função da influência das cuestas $^{14}$, o benefício foi moderadamente menos valorizado pela população, em Bauru, onde as temperaturas são mais elevadas, foi moderadamente mais valorizado. Um outro fator específico observado e que se relaciona com a valorização de um benefício é a adoção prioritária de medidas e políticas que valorizam os benefícios ambientais em Botucatu. A moderada valorização do benefício "contribuir para a biodiversidade", bem como a valorização, no conjunto, dos benefícios ambientais pode estar relacionada às ações de Organizações Não-Governamentais (ONGs) juntamento com a população.

Além dos fatores específicos relacionados aos contextos político, social e urbano de cada município, observa-se que contextos globais também surgem em meio a classificação. Em uma breve comparação com alguns estudos realizados em diferentes partes do mundo, constata-se que a valorização do benefício "promover a saúde e bem-estar" como o mais

\footnotetext{
${ }^{14}$ Cuesta de Botucatu: Área de transição entre a Depressão Periférica e o Planalto Ocidental, a Cuesta Basáltica apresenta vertentes muito íngremes na direção da depressão e outras mais acentuadas na direção do Planalto. O município de Botucatu apresenta três regiões distintas: "Baixada", correspondente à Depressão Periférica; "Topo da Cuesta", pertencente ao Planalto Ocidental Paulista; e a "Frente da Cuesta", uma zona de transição entre essas duas regiões. A sede do município localiza-se no Topo da Cuesta, com altitudes que variam de 756 a 920m [...].". (Maroni, Si Stasi e Machado, 2006, P. 12).
} 
importante assemelha-se à resposta dos participantes dos estudos de Portugal (Madureira et al, 2014) e China (Jim e Shan, 2013), refletindo uma tendência global atual de cuidado com a mente e o corpo, que vai além da cultura e características locais. Ademais, "diminuir a poluição do ar da cidade", além de acompanhar parcialmente o estudo português (Madureira et al., 2014), assemelha-se à pesquisa realizada no Canadá (Peckham, Duinker e Ordóñez, 2013), seguindo novamente uma preocupação global em relação à emissão de poluentes. Entre os menos valorizados, constata-se uma semelhança com os resultados obtidos por Madureira et al. (2014) para os benefícios "atenuar o ruído na cidade" e "facilitar o convívio e a interação social", expressando, este último, a dificuldade do Homem contemporâneo de conviver nos espaços livres urbanos, pois a sensação de (in)segurança altera a forma de utilização dos espaços.

\section{Conclusões}

Os diversos benefícios dos espaços verdes urbanos têm incentivado uma abordagem multifuncional por parte dos cidadãos e pesquisadores, contudo tem desafiado a ação de administradores e planejadores. Assim, com a presente pesquisa constatou-se que um dos caminhos para o reconhecimento dos benefícios destes espaços está em estudos baseados em análises multimétodos, que ocorrem em conjunto com profissionais e a população. A complementaridade entre diversas pesquisas que focam nos contextos histórico, político, social e urbano proporcionam um conjunto de informações fundamentais para as tomadas de decisão.

A aplicação do método Best-Worst Scaling com a população de Botucatu e Bauru traz uma nova proposta de pesquisa junto aos cidadãos, propiciando uma visão hierárquica dos benefícios dos espaços verdes urbanos. Apesar dos benefícios não serem igualmente valorizados pelos participantes, observa-se que existe um consenso entre os mais e menos importantes. Pode-se afirmar que são diversos os fatores responsáveis pelas escolhas da população. Assim, apesar da ampla abordagem sobre as características socioambientais e as políticas públicas municipais para os espaços verdes, novas esferas de abordagem devem ser utilizadas em estudos futuros, para verificar a relação de influência dos benefícios sobre 
a população, já que as especificidades socioambientais e políticas levantadas não se mostraram fatores únicos para diferenciar a hierarquização dos cidadãos.

No entanto, observa-se que os fatores socioambientais e políticos interferem diretamente na percepção dos cidadãos. Há, entre as duas cidades, uma absoluta diferença na frequência de visitas a jardins, na satisfação com os espaços verdes e na escolha dos benefícios, quando considerado o conjunto de atributos. O uso e a satisfação com os espaços verdes da cidade e a valorização de benefícios ambientais, no seu conjunto, por parte dos cidadãos entrevistados em Botucatu, revela o reconhecimento da importância desses locais para a qualidade do meio urbano e das ações públicas municipais desenvolvidas, apresentando um consenso entre as esferas pública e popular. As particularidades locais, como a inserção do município na Cuesta de Botucatu e na Área de Preservação Ambiental (APA), parecem interferir de forma positiva no reconhecimento dos benefícios e da importância desses espaços. Já a baixa frequência de visitas a jardins e parques públicos, a insatisfação com os espaços verdes urbanos da cidade e próximos da residência, e a valorização dos benefícios sociais e culturais por parte dos cidadãos de Bauru, constituem um reflexo negativo das políticas e ações adotadas pela gestão municipal. Constata-se, portanto, que há, entre as duas cidades, uma absoluta diferença: enquanto em Botucatu há um consenso entre as esferas pública e popular, em Bauru, há um contraste.

Segundo Madureira et al. (2015), estudos dessa natureza, como o realizado nas duas cidades do oeste-paulista, trazem duas importantes compreensões sobre a infraestrutura verde e a participação popular nas tomadas de decisão. A primeira é sobre a real necessidade de se considerar a opinião dos cidadãos sobre os espaços verdes, a fim de incentivar avaliações locais e evitar, dessa maneira, ações e medidas generalistas. A segunda é a necessidade de comunicação entre planejadores e pesquisadores sobre os benefícios disponibilizados pela infraestrutura verde urbana, uma vez que esses não são igualmente reconhecidos entre diferentes cidades, evitando, dessa forma, possíveis conflitos com a população.

O processo de construção de uma nova gestão ambiental ainda está sendo construído em Botucatu, assim como os conceitos contemporâneos de paisagem e de valorização dos espaços verdes no meio urbano; portanto, é fundamental o desenvolvimento de ações 
conscientes e trabalho conjunto. No levantamento das principais legislações ficou evidente o reconhecimento dos condicionantes físicos da paisagem pelo poder público de Bauru, principalmente com as mudanças e tendências de valorização dos espaços verdes urbanos, contudo, apenas conceitos e ideias não são suficientes para construir um ambiente de qualidade. O que falta, agora, no munícipio, são ações práticas, eficientes, que transformem em realidade todos os conceitos e ideais desenvolvidos pelas gestões passadas. Como podese observar, as cidades, assim como as pessoas, estão em constante processo de mudança e transformação. Acompanhá-las é fundamental para as tomadas de decisão e de valorização dos espaços verdes.

Reitera-se, que, além de um aprofundamento no estudo realizado nos municípios de Botucatu e Bauru, pesquisas semelhantes em outras áreas urbanas, nacionais e internacionais, são fundamentais para ampliar a base comparativa e abordar com maior detalhamento as interferências dos contextos sociais, culturais, políticos, históricos, ambientais e territoriais nas tomadas de decisão de cidadãos, planejadores e gestores para uma conformação da paisagem e um ordenamento das cidades de qualidade.

\section{Referências bibliográficas}

BAYCAN-LEVENT, Tuzin; VREEKER Ron and NIJKAMP, Peter. A Multi-Criteria evaluation of green spaces in European cities. European Urban and Regional Studies, 2009, vol. 16, $n^{\circ} 2$ [consultado 24 de abril de 2015], p. 193-213. Disponível em: <http://eur.sagepub.com/content/16/2/193.abstract>.

BURKE, Paul F.; LOUVIERE, Jordan; WEI, Edward; MACAULAY, Gordon; QUAIL, Ken and CARSON, Richard. Overcoming challenges and improvements in Best-Worst Elicitation: Determining what matters to Japanese Wheat Millers. In International Choice Modelling Conference 2013. Open Conference System, 2013 [consultado 20 de novembro de 2014]. Disponível em: <http://www.icmconference.org.uk/index.php/icmc/ICMC2013/paper/view/615>.

CARMAN, Nancy. Introduction to the LATIS Forum on Therapeutic Gardens. In LATIS FORUM ON THERAPEUTIC GARDENS, Washington, 2005. Landscape architecture technical information series. Washington: American Society of Landscape Architects, 2005.

CASINI, Leonardo; CORSI, Armando Maria; LOCKSHIN, Larry; COHEN, Eli and GOODMAN, Steve. Consumer preferences of wine in Italy applying best-worst scaling. International Journal of Wine Business Research, 2008, vol. $21, n^{\circ} 1$, p. 64-78.

CUNHA, Daniel Oliveira. O Processo de Decisão de Compra de Vinho: Uma análise comparativa dos consumidores cariocas, paulistas e portugueses. Dissertação de Mestrado em Marketing, Faculdade de Economia, Universidade do Porto, Porto, 2013. [Consultado 24 de junho de 2015]. Disponível em: < sigarra.up.pt/fep/pt/publs_pesquisa.show_publ_file?pct_gdoc_id=63232>.

DE MAGISTRIS, Tiziana; GROOT, Etiénne; GRACIA, Azucena and ALBISU, Luis Miguel. Consumers preferences for wine in Spain: best-worst scaling methodology. In EAAE 2011 INTERNATIONAL CONGRESS, 2011, Zurich. 
Challenges for Agriculture, Food and Natural Resources. Zurich, 2011 [consultado 20 de novembro de 2014]. Disponível em: < http://ageconsearch.umn.edu/bitstream/114449/2/de\%20Magistris_Tiziana_211.pdf>.

DINES, Nicholas; CATTELL, Vicky; GESLER, Wil and CURTIS, Sarah. Public Spaces, social relations and well-being in East London. The Policy Press, Great Britain. 2006.

FÁLCON, Antoni. Espacios verdes para una ciudad sostenible. Planificación, proyecto, mantenimiento y gestión. Barcelona: Editorial Gustavo Gili, 2007.

FLYNN, T. N. and MARLEY, A. A. J. Best Worst Scaling: Theory and Methods. Handbook of Choice Modelling, 2014. [consultado 24 de junho de 2015]. Disponível em: < https://www.unisa.edu.au/Global/business/centres/i4c/docs/papers/wp12-002.pdf >.

FORMAN, Richard T. Land Mosaics: the ecology of landscape and regions. New York: Cambridge Press, 1995.

HERZOG, Cecilia Polacow. Cidade para todos: re-aprendendo a conviver com a natureza. Rio de Janeiro: Mauad X/ Inverde, 2013.

HOUGH, Michael. Naturaleza y ciudad: planificación urbana y processos ecológicos. Barcelona: Editora Gustavo Gili, S. A., 1998. ISBN:84-252-1632-X.

INSTITUTO BRASILEIRO DE GEOGRAFIA E ESTATÍSTICA - IBGE. Portal SIDRA. Censo 2010. Disponível em:< http://www.sidra.ibge.gov.br/bda/tabela/listabl.asp?c=3533\&z=cd\&o=16>.

JACOBS, Jane. Morte e Vida de Grandes Cidades. Tradução: Carlos S. Mendes Rosa. São Paulo: Martins Fontes, 2000. ISBN:85-336-1218-4.

JIM, C. Y.; SHAN, X. Socioeconomic effect on perception of urban green spaces in Guangzhou, China. Cities, 2013, vol. 31 [Consultado 24 de abril de 2015], p. 123-131. Disponível em: < http://www.journals.elsevier.com/cities/>.

KAPLAN, Stephen. The restorative benefits of nature: Toward an integrative framework. Journal of Environmental Psychology, 1995, vol. 15, n³, p.169-182.

KAPLAN, Rachel. The nature of the view from home: Psychological Benefits. Environment and behavior, 2001, vol. 33, n 4, p.507-542.

LEITE, Maria Angela Faggin Pereira. Projeto e uso dos espaços públicos, o código e a interpretação. In Mesa Redonda Representações da Cidade: Imagens Cruzadas entre Brasil e Europa: atas do III Congresso da Brasilian Studies Association, Cambridge, 1996. Cambridge, Reino Unido, 1996, p. 01-15.

MADUREIRA, Helena; NUNES, Fernando; OLIVEIRA, José Vidal and MADUREIRA, Teresa and CORMIER; L. Benefícios atribuídos aos espaços verdes urbanos pela população: resultados de um inquérito conduzido em Lisboa e no Porto. In PLURIS14 (Re) inventar a cidade em tempos de mudança: livro de actas, 2014, Lisboa. Lisboa, 2014.

MADUREIRA, Helena; NUNES, Fernando; OLIVEIRA, José Vidal and MADUREIRA, Teresa. Urban resident's beliefs concerning green space benefits in four cities in France and Portugal. Urban Forestry \& Urban Greening, 2015, vol.14, p. 56-64.

MARONI, Beatriz Castro; DI STASI, Luiz Claudio and MACHADO, Silvia Rdrigues. Plantas medicinais do cerrado de Botucatu - Guia Ilustrado. São Paulo: Editora UNESP, 2006.

MATTOS, Karina Andrade. Espaços verdes urbanos: análise multimétodos para a valorização. Dissertação de Mestrado em Arquitetura e Urbanismo, Faculdade de Arquitetura, Artes e Comunicação, Universidade Estadual Paulista "Júlio de Mesquita Filho", Bauru, $2017 . \quad$ Disponível em:<https://repositorio.unesp.br/handle/11449/149998>.

MAZZEI, Katia; COLESANTI, Marlene T. M.; SANTOS, Douglas Gomes. Áreas verdes urbanas, espaços livres para lazer. Sociedade \& Natureza, 2007, vol.19, $n^{\circ} 1$ [consultado 23 de outubro de 2014] p. 33-43. Disponível em:< www.seer.ufu.br/index.php/sociedadenatureza/article/view/9350/5730>.

MOORE, Robin C. and COSCO, Nilda G. Well-being by Nature: Therapeutic Gardens for Children. In LATIS FORUM ON THERAPEUTIC GARDENS, Washington, 2005. Landscape architecture technical information series. Washington: American Society of Landscape Architects, 2005, p. 35-50. 
PECKHAM, Shawna C.; DUINKER, Peter N.; ORDÓÑEZ, Camilo. Urban forest values in Canada: Views of citizens in Calgary and Halifax. Urban Forestry \& Urban Greening. 2013, vol. 12 [Consultado 24 de abril de 2015], p. 154-162. Disponível em: <http://www.sciencedirect.com/science/article/pii/S1618866713000034>.

PELLEGRINO, Paulo Renato Mesquita; GUEDES, Paula Pinto; PIRILLO, Fernanda Cunha Pirillo and FERNANDES, Sávio Almeida. A paisagem da borda: uma estratégia para a condução das águas, da biodiversidade e das pessoas. In COSTA, Lúcia Maria Sá Antunes (Org.). Rios e paisagens urbanas em cidades brasileiras. Rio de Janeiro: Viana \& Mosley Editora PROURB, 2006, p.57-75. ISBN:85-88721-38-4.

ROMERO, Marta Adriana Bustos. A arquitetura bioclimática do espaço público. Brasília: Editora Universidade de Brasília, 2001. ISBN:85-230-0652-4.

ROMERO, Marta Adriana Bustos. Arquitetura Bioclimática dos Espaços Públicos. Brasília: Editora UnB, 2007.

SANESI, Giovanni and CHIARELLO, Francesco. Residents and urban green spaces: The case of Bari. Urban Forestry \& Urban Greening. 2006, vol. 4 [Consultado 24 de abril de 2015], p.125-134. Disponível em: <http://www.sciencedirect.com/science/article/pii/S1618866705000531>.

SAWTOOTH SOFTWARE. The MaxDiff System Technical Paper. Orem, Utah: Sawtooth Software Inc., 2013. [Consultado 27 de abril de 2015]. Disponível em:< http://www.sawtoothsoftware.com/support/technicalpapers/maxdiff-best-worst-scaling/maxdiff-technical-paper-2013>.

SPIRN, Anne Whiston. O Jardim de Granito: A Natureza no Desenho da Cidade. Tradução de Paulo Renato Mesquita Pellegrino. São Paulo: Editora da Universidade de São Paulo, 1995. ISBN:85-314-0158-5.

THOMPSON, Catharine Ward. Playful Nature: What makes the difference between some people going outside and others not?. In THOMPSON, C. W.; TRAVLOU, P. Open Space: People Space. Taylor \& Francis, 2007, p. 2637.

VESELY, Éva-Terézia. Green for green: The perceived value of a quantitative change in the urban tree estate of New Zealand. Ecological Economics, 2007, vol. 63 [Consultado 24 de abril de 2015] p. 605-615. Disponível em: $<$ www.elsevier.com/locate/ecolecon>. 